\title{
The major arcs approximation of an exponential sum over primes
}

by

D. A. Goldston (San Jose, CA)

1. Introduction. Let

$$
S(\alpha)=\sum_{n \leq N} \Lambda(n) e(n \alpha), \quad e(u)=e^{2 \pi i u},
$$

where $\Lambda(n)$ is the von Mangoldt function defined by $\Lambda(n)=\log p$ if $n=p^{m}$, $m \geq 1, p$ a prime, and $\Lambda(n)=0$ otherwise. Parseval's equation and the prime number theorem provide the $L^{2}$ estimate

$$
\int_{0}^{1}|S(\alpha)|^{2} d \alpha=\sum_{n \leq N} \Lambda^{2}(n) \sim N \log N \quad \text { as } N \rightarrow \infty,
$$

and therefore by the Cauchy-Schwarz inequality we have, for any $\varepsilon>0$ and $N$ sufficiently large,

$$
\int_{0}^{1}|S(\alpha)| d \alpha \leq(1+\varepsilon) \sqrt{N \log N}
$$

In this paper we obtain the following improvement on this $L^{1}$ upper bound.

TheOREM. For any $\varepsilon>0$ and $N$ sufficiently large we have

$$
\int_{0}^{1}|S(\alpha)| d \alpha \leq\left(\frac{\sqrt{2}}{2}+\varepsilon\right) \sqrt{N \log N} .
$$

Vaughan [3] has shown that

$$
\int_{0}^{1}|S(\alpha)| d \alpha \gg \sqrt{N}
$$

2000 Mathematics Subject Classification: Primary 11L20.

Research supported in part by NSERC Grant A5123 and NSF grants. 
and has also made the conjecture that there exists a constant $c$ such that, as $N \rightarrow \infty$,

$$
\int_{0}^{1}|S(\alpha)| d \alpha \sim c \sqrt{N \log N} .
$$

This conjecture may be very difficult to prove, but it might be possible to obtain the lower bound

$$
\int_{0}^{1}|S(\alpha)| d \alpha \gg \sqrt{N \log N} .
$$

I have proved that (1.7) follows from a strong form of either the Goldbach or the twin prime conjectures. Further, certain approximations of $S(\alpha)$ also satisfy (1.7). These results will be presented in a later paper.

Notation. We use the following notation. We take $n, q, r, k, j$ to always be positive integers, and in general summation signs will start with 1 if a lower limit is not specified. We will use the notation

$$
\sum_{a(q)}^{*}=\sum_{\substack{1 \leq a \leq q \\(a, q)=1}} \text { and } \sum_{Q}=\sum_{1 \leq q \leq Q} \sum_{a(q)}^{*} .
$$

2. The major arc approximation. Hardy and Littlewood ([1], [2]) introduced the method used for the analysis of $S(\alpha)$. For $\alpha$ near a fraction $a / q, S(\alpha)=S(a / q+\beta)$ is large and can be approximated well by the simple expression

$$
\frac{\mu(q)}{\phi(q)} I(\beta)
$$

where

$$
I(\beta)=\sum_{n=1}^{N} e(n \beta) .
$$

Here $\alpha=a / q+\beta$ where $\beta$ needs to be small so that there are no other fractions with denominators $\leq q$ within a distance of $|\beta|$ of $a / q$. This requirement leads to the Farey decomposition of the unit interval. The Farey fractions of order $Q$ are given by

$$
\mathcal{F}_{Q}=\{a / q: 1 \leq q \leq Q, 0 \leq a \leq q,(a, q)=1\} .
$$

We define the Farey arcs around each of these fractions, except $0 / 1$ which we exclude, as follows. Let $a^{\prime} / q^{\prime}<a / q<a^{\prime \prime} / q^{\prime \prime}$ be consecutive fractions in 
the Farey decomposition of order $Q$, and let

$$
\begin{aligned}
& \mathcal{M}_{Q}(q, a)=\left(\frac{a+a^{\prime}}{q+q^{\prime}}, \frac{a+a^{\prime \prime}}{q+q^{\prime \prime}}\right] \quad \text { for } \frac{a}{q} \neq \frac{1}{1}, a \neq 0, \\
& \mathcal{M}_{Q}(1,1)=\left(1-\frac{1}{Q+1}, 0\right] \cup\left(0, \frac{1}{Q+1}\right] .
\end{aligned}
$$

These intervals are disjoint and their union covers the interval $[0,1]$. We will sometimes shift these intervals to the origin, which we denote by

$$
\theta_{Q}(q, a)=\left(\frac{a+a^{\prime}}{q+q^{\prime}}-\frac{a}{q}, \frac{a+a^{\prime \prime}}{q+q^{\prime \prime}}-\frac{a}{q}\right]=\left(\frac{-1}{q\left(q+q^{\prime}\right)}, \frac{1}{q\left(q+q^{\prime \prime}\right)}\right]
$$

when $q \neq 1$, and $\theta_{Q}(1,1)=(-1 /(Q+1), 1 /(Q+1)]$. Since $Q<q+q^{\prime}<2 Q$ and similarly for $q+q^{\prime \prime}$, we see

$$
\theta_{Q}(q, a)=\left(-\frac{1}{q(Q+\mu)}, \frac{1}{q(Q+\nu)}\right],
$$

for integers $0<\mu, \nu<Q$ which depend on $a, q$, and $Q$. In particular,

$$
\left(\frac{-1}{2 q Q}, \frac{1}{2 q Q}\right) \subseteq \theta_{Q}(q, a) \subseteq\left(\frac{-1}{q Q}, \frac{1}{q Q}\right) .
$$

Finally we define the characteristic function of $\mathcal{M}_{Q}(q, a)$ by

$$
\chi_{Q}(\alpha ; q, a)= \begin{cases}1 & \text { if } \alpha \in \mathcal{M}_{Q}(q, a), \\ 0 & \text { otherwise. }\end{cases}
$$

With this preparation, our approximation to $S(\alpha)$ is given by

$$
J_{Q}(\alpha)=\sum_{1 \leq q \leq Q} \sum_{a(q)}^{*} \frac{\mu(q)}{\phi(q)} I\left(\alpha-\frac{a}{q}\right) \chi_{Q}(\alpha ; q, a) .
$$

We will call $J_{Q}(\alpha)$ the major arcs approximation for $S(\alpha)$. This approximation has the advantage that each term in the approximation is orthogonal to every other on the unit interval, which greatly simplifies the computation of various means. One expects that $J_{Q}(\alpha)$ will become a better approximation of $S(\alpha)$ as $Q$ is increased as a function of $N$. For the $L^{2}$ norm this is the case when $Q \leq \sqrt{N}$, but for $\sqrt{N}<Q \leq N$ the approximation degrades because the support $\chi_{Q}(\alpha ; q, a)$ becomes so small that the terms with $q \geq N / Q$ no longer make any contribution. Because of this we define

$$
Q_{1}=\min (Q, N / Q)= \begin{cases}Q & \text { if } 1 \leq Q \leq \sqrt{N} \\ N / Q & \text { if } \sqrt{N} \leq Q \leq N\end{cases}
$$

and we interpret $J_{Q}(\alpha)$ as actually being an $L^{2}$ approximation to $S(\alpha)$ of length $Q_{1}$ rather than $Q$. This disadvantage of $J_{Q}(\alpha)$ may be corrected by simply deleting the factor $\chi_{Q}(\alpha ; q, a)$ in the definition of $J_{Q}(\alpha)$, but the resulting approximation is much harder to analyze when $Q>\sqrt{N}$. 
We comment that the term "major arcs" has different meanings depending on the problem the circle method is being applied to as well as individual taste. Our function $J_{Q}(\alpha)$ is not the only possible choice for an approximation. One complication in applying $J_{Q}(\alpha)$ is that in some situations one needs to take account of the exact positions of the endpoints of the Farey arcs, which introduces Kloosterman sums. This can often be avoided by using arcs that do not depend on $a$, such as the intervals in (2.5) that envelope $\theta_{Q}(q, a)$ or sometimes even intervals that do not depend on $q$. In this paper however there is no problem with using the Farey arcs in our approximation.

The idea for the proof of the Theorem is quite simple. We write

$$
S(\alpha)=J_{Q}(\alpha)+T_{Q}(\alpha),
$$

and refer to $T_{Q}(\alpha)$ defined by $(2.9)$ as the minor arcs part of $S(\alpha)$. The major arcs approximation $J_{Q}(\alpha)$ consists of spikes which are amplified when we take higher powers. As we will see in the next section, $J_{Q}(\alpha)$ makes only a contribution of at most $\sqrt{N}$ in the $L^{1}$ norm, but on squaring it contributes half of the amount on the right hand side of (1.2) when $Q=\sqrt{N}$. Further, in Section 4 we show $J_{Q}(\alpha)$ and $T_{Q}(\alpha)$ are essentially orthogonal in $L^{2}$, so that $T_{Q}(\alpha)$ contributes the other half of the amount in (1.2). Thus, in $L^{1}$ the size of $S(\alpha)$ is determined by $T_{Q}(\alpha)$, which satisfies an $L^{2}$ bound one half as large as the bound in (1.2).

3. Means of $J_{Q}(\alpha)$. We prove in this section some results on means of $J_{Q}(\alpha)$.

Lemma 1 . For $1 \leq Q \leq N$ and $Q_{1}=\min (Q, N / Q)$, we have

$$
\int_{0}^{1}\left|J_{Q}(\alpha)\right| d \alpha \asymp Q_{1} \log \frac{2 N}{Q_{1}^{2}}
$$

In particular,

$$
\int_{0}^{1}\left|J_{Q}(\alpha)\right| d \alpha \ll \sqrt{N}
$$

Lemma 2. For $1 \leq Q \leq N, Q_{1}=\min (Q, N / Q)$, and $k$ a positive integer, we have

$$
\int_{0}^{1}\left|J_{Q}(\alpha)\right|^{2 k} d \alpha=\left(\sum_{q \leq Q_{1}} \frac{\mu^{2}(q)}{\phi^{2 k-1}(q)}\right)\left(\sum_{n \leq k N} \nu_{k}^{2}(n)\right)+O\left(Q^{2 k-1} Q_{1}\right),
$$

where

$$
\nu_{k}(n)=\nu_{k}(n, N)=\sum_{\begin{array}{c}
n_{1}, \ldots, n_{k} \leq N \\
n_{1}+\ldots+n_{k}=n
\end{array}} 1 .
$$

In particular, for $k=1$ we have 


$$
\int_{0}^{1}\left|J_{Q}(\alpha)\right|^{2} d \alpha=N \log Q_{1}+O(N) .
$$

Pro of (of Lemma 1). By (2.7),

$$
\begin{aligned}
\int_{0}^{1}\left|J_{Q}(\alpha)\right| d \alpha & =\sum_{Q} \frac{\mu^{2}(q)}{\phi(q)} \int_{\mathcal{M}_{Q}(q, a)}\left|I\left(\alpha-\frac{a}{q}\right)\right| d \alpha \\
& =\sum_{Q} \frac{\mu^{2}(q)}{\phi(q)} \int_{\theta_{Q}(q, a)}|I(\beta)| d \beta .
\end{aligned}
$$

Since

we have, by (2.5),

$$
I(\beta)=e\left(\frac{(N+1) \beta}{2}\right) \frac{\sin (\pi N \beta)}{\sin (\pi \beta)},
$$

$$
\begin{aligned}
\int_{\theta_{Q}(q, a)}|I(\beta)| d \beta & =\int_{\theta_{Q}(q, a)}\left|\frac{\sin (\pi N \beta)}{\sin (\pi \beta)}\right| d \beta \\
& =2 \int_{0}^{1 /(q Q)}\left|\frac{\sin (\pi N \beta)}{\sin (\pi \beta)}\right| d \beta+O(1) .
\end{aligned}
$$

Using

$$
\begin{aligned}
\int_{0}^{1 /(q Q)}\left|\frac{\sin (\pi N \beta)}{\sin (\pi \beta)}\right| d \beta & \asymp \int_{0}^{N /(q Q)}\left|\frac{\sin (\pi u)}{u}\right| d u \\
& \asymp \begin{cases}N /(q Q) & \text { if } N /(q Q) \leq 1, \\
\log ((2 N) /(q Q)) & \text { if } N /(q Q) \geq 1,\end{cases}
\end{aligned}
$$

together with (3.6) and (3.7), for $Q \leq N^{1 / 2}$ we have

$$
\begin{aligned}
\int_{0}^{1}\left|J_{Q}(\alpha)\right| d \alpha & \asymp \sum_{q \leq Q} \mu^{2}(q)\left(\int_{0}^{1 /(q Q)}\left|\frac{\sin (\pi N \beta)}{\sin (\pi \beta)}\right| d \beta+O(1)\right) \\
& \asymp \sum_{q \leq Q} \mu^{2}(q) \log \frac{2 N}{q Q} \asymp Q \log \frac{2 N}{Q^{2}}=Q_{1} \log \frac{2 N}{Q_{1}^{2}},
\end{aligned}
$$

since $Q_{1}=Q$ in this case, while for $Q>N^{1 / 2}$,

$$
\begin{aligned}
\int_{0}^{1}\left|J_{Q}(\alpha)\right| d \alpha & \asymp \sum_{q \leq Q_{1}} \mu^{2}(q) \log \frac{2 N}{q Q}+\sum_{Q_{1}<q \leq Q} \mu^{2}(q) \frac{N}{q Q} \\
& \asymp Q_{1} \log \frac{2 N}{Q_{1} Q}+\frac{N}{Q} \log \frac{Q}{Q_{1}} \asymp Q_{1} \log \frac{2 Q}{Q_{1}}=Q_{1} \log \frac{2 N}{Q_{1}^{2}},
\end{aligned}
$$

which completes the proof of the lemma. 
Pro of (of Lemma 2). We have

$$
\int_{0}^{1}\left|J_{Q}(\alpha)\right|^{2 k} d \alpha=\sum_{Q} \frac{\mu^{2}(q)}{\phi^{2 k}(q)} \int_{\mathcal{M}_{Q}(q, a)}\left|I\left(\alpha-\frac{a}{q}\right)\right|^{2 k} d \alpha .
$$

Now

$$
\begin{aligned}
& \int_{\mathcal{M}_{Q}(q, a)}\left|I\left(\alpha-\frac{a}{q}\right)\right|^{2 k} d \alpha \\
& =\int_{\theta_{Q}(q, a)}|I(\beta)|^{2 k} d \beta=\int_{0}^{1}|I(\beta)|^{2 k} d \beta-\int_{[0,1] \backslash \theta_{Q}(q, a)}|I(\beta)|^{2 k} d \beta \\
& =\int_{0}^{1}\left|\sum_{n \leq k N} \nu_{k}(n) e(n \beta)\right|^{2} d \beta+O\left(\int_{1 /(2 q Q)}^{1 / 2} \frac{1}{\beta^{2 k}} d \beta\right) \\
& =\sum_{n \leq k N} \nu_{k}^{2}(n)+O\left((q Q)^{2 k-1}\right) .
\end{aligned}
$$

We also have the trivial bound

$$
\int_{\mathcal{M}_{Q}(q, a)}\left|I\left(\alpha-\frac{a}{q}\right)\right|^{2 k} d \alpha \ll \frac{N^{2 k}}{q Q}
$$

which becomes smaller than the error in (3.9) provided $q \geq N / Q$. We conclude

$$
\begin{aligned}
\int_{0}^{1}\left|J_{Q}(\alpha)\right|^{2 k} d \alpha= & \sum_{q \leq Q_{1}} \frac{\mu^{2}(q)}{\phi^{2 k}(q)} \sum_{a(q)}^{*}\left(\sum_{n \leq k N} \nu_{k}^{2}(n)+O\left((q Q)^{2 k-1}\right)\right) \\
& +O\left(\frac{N^{2 k}}{Q} \sum_{Q_{1}<q \leq Q}^{\prime} \frac{\mu^{2}(q)}{q \phi^{2 k-1}(q)}\right) \\
= & \left(\sum_{q \leq Q_{1}} \frac{\mu^{2}(q)}{\phi^{2 k-1}(q)}\right)\left(\sum_{n \leq k N} \nu_{k}^{2}(n)\right) \\
& +O\left(Q^{2 k-1} Q_{1}\right)+O^{\prime}\left(\frac{N^{2 k}}{Q Q_{1}^{2 k-1}}\right),
\end{aligned}
$$

where the dash indicates this term only occurs if $Q>N^{1 / 2}$. We thus see that for $Q \leq N^{1 / 2}$ the error term above is $\ll Q^{2 k-1} Q_{1}$ as stated in Lemma 2, while for $Q>N^{1 / 2}$ the error is $\ll N Q^{2 k-2}=Q^{2 k-1} Q_{1}$ and the lemma follows. In the case $k=1$ the result follows from the well known formula [4]

$$
\sum_{q \leq Q} \frac{\mu^{2}(q)}{\phi(q)}=\log Q+O(1)
$$


4. Inner product of $J_{Q}(\alpha)$ and $S(\alpha)$. The pointwise behaviour of $S(\alpha)$ is complicated and the known results are much weaker than what is expected to be true. However when one averages $S(\alpha)$ over the reduced fractions with denominator $q$ one easily obtains an asymptotic formula. It is useful to obtain a result of this type due to Vaughan who used it in proving (1.5).

LEMma 3. We have

$$
\sum_{a(q)}^{*} S\left(\frac{a}{q}+\beta\right)=\mu(q) S(\beta)+O(\phi(q) \log (2 N) \log (2 q)) .
$$

Proof. We have

$$
\sum_{a(q)}^{*} S\left(\frac{a}{q}+\beta\right)=\sum_{n \leq N} \Lambda(n) e(n \beta) \sum_{a(q)}^{*} e\left(\frac{a n}{q}\right)=\sum_{n \leq N} \Lambda(n) c_{q}(n) e(n \beta),
$$

where $c_{q}(n)$ is Ramanujan's sum. Since $c_{q}(n)=\mu(q)$ if $(n, q)=1$, and trivially $\left|c_{q}(n)\right| \leq \phi(q)$, we have

$$
\begin{aligned}
\sum_{n \leq N} \Lambda(n) c_{q}(n) e(n \beta) & =\mu(q) \sum_{\substack{n \leq N \\
(n, q)=1}} \Lambda(n) e(n \beta)+O\left(\sum_{\substack{n \leq N \\
(n, q)>1}} \Lambda(n) \phi(q)\right) \\
& =\mu(q) S(\beta)+O\left(\phi(q) \sum_{\substack{n \leq N \\
(n, q)>1}} \Lambda(n)\right) \\
& =\mu(q) S(\beta)+O(\phi(q) \log (2 N) \log (2 q)) .
\end{aligned}
$$

We next evaluate the inner product of $S(\alpha)$ and $J_{Q}(\alpha)$.

Lemma 4. For $1 \leq Q \leq N$ and $Q_{1}=\min (Q, N / Q)$, we have

$$
\int_{0}^{1} S(\alpha) \overline{J_{Q}(\alpha)} d \alpha=N \log Q_{1}+O(N \sqrt{\log (2 N)}) .
$$

Proof. We have

$$
\begin{aligned}
\int_{0}^{1} S(\alpha) \overline{J_{Q}(\alpha)} d \alpha & =\sum_{Q} \frac{\mu(q)}{\phi(q)} \int_{\theta_{Q}(q, a)} S\left(\frac{a}{q}+\beta\right) \overline{I(\beta)} d \beta \\
& =\sum_{q \leq Q_{1}}+\sum_{Q_{1}<q \leq Q}=S_{1}+S_{2} .
\end{aligned}
$$

For the main term $S_{1}$ we have

$$
S_{1}=\sum_{q \leq Q_{1}} \frac{\mu(q)}{\phi(q)} \sum_{a(q)}^{*} \int_{\theta_{Q}(q, a)} S\left(\frac{a}{q}+\beta\right) \overline{I(\beta)} d \beta
$$




$$
\begin{aligned}
= & \sum_{q \leq Q_{1}} \frac{\mu(q)}{\phi(q)} \sum_{a(q)}^{*} \int_{0}^{1} S\left(\frac{a}{q}+\beta\right) \overline{I(\beta)} d \beta \\
& -\sum_{q \leq Q_{1}} \frac{\mu(q)}{\phi(q)} \sum_{a(q)}^{*} \int_{[0,1] \backslash \theta_{Q}(q, a)} S\left(\frac{a}{q}+\beta\right) \overline{I(\beta)} d \beta \\
= & S_{3}-S_{4} .
\end{aligned}
$$

Now

$$
\begin{aligned}
\int_{0}^{1} S\left(\frac{a}{q}+\beta\right) \overline{I(\beta)} d \beta & =\sum_{n \leq N} \Lambda(n) e\left(\frac{n a}{q}\right) \int_{0}^{1} e(n \beta) \overline{I(\beta)} d \beta \\
& =\sum_{n \leq N} \Lambda(n) e\left(\frac{n a}{q}\right)=S\left(\frac{a}{q}\right),
\end{aligned}
$$

and therefore by Lemma 3,

$$
\begin{aligned}
S_{3} & =\sum_{q \leq Q_{1}} \frac{\mu(q)}{\phi(q)} \sum_{a(q)}^{*} S\left(\frac{a}{q}\right) \\
& =\sum_{q \leq Q_{1}} \frac{\mu^{2}(q)}{\phi(q)} S(0)+O\left(\sum_{q \leq Q_{1}} \mu^{2}(q) \log (2 N) \log (2 q)\right) \\
& =\psi(N) \sum_{q \leq Q_{1}} \frac{\mu^{2}(q)}{\phi(q)}+O\left(Q_{1} \log ^{2}(2 N)\right),
\end{aligned}
$$

where $\psi(x)=\sum_{n \leq x} \Lambda(n)$. By the prime number theorem $\psi(x)=x+$ $O\left(x(\log x)^{-A}\right)$ for any $A>0$. Hence, by (3.11),

$$
\begin{aligned}
S_{3} & =\left(N+O\left(\frac{N}{\log ^{A} N}\right)\right) \sum_{q \leq Q_{1}} \frac{\mu^{2}(q)}{\phi(q)}+O\left(Q_{1} \log ^{2}(2 N)\right) \\
& =N \log Q_{1}+O(N) .
\end{aligned}
$$

To estimate $S_{4}$, we have

$$
\begin{aligned}
S_{4} & =\sum_{q \leq Q_{1}} \frac{\mu(q)}{\phi(q)} \sum_{a(q)}^{*}\left(\int_{1 /(2 q Q)}^{1-1 /(2 q Q)}-\int_{\mathcal{N}}\right) S\left(\frac{a}{q}+\beta\right) \overline{I(\beta)} d \beta \\
& =S_{5}-S_{6},
\end{aligned}
$$

where

$$
\mathcal{N}=\left[\frac{1}{2 q Q}, \frac{1}{q(Q+\nu)}\right] \cup\left[-\frac{1}{q(Q+\mu)},-\frac{1}{2 q Q}\right] \subset \theta_{Q}(q, a)
$$

where $\nu$ and $\mu$ are the numbers in (2.4) which depend on $a, q$, and $Q$. By 
Cauchy-Schwarz we have

$$
\begin{aligned}
S_{6} \leq & \sum_{q \leq Q_{1}} \frac{\mu^{2}(q)}{\phi(q)} \sum_{a(q)}^{*}\left(\int_{\mathcal{N}}\left|S\left(\frac{a}{q}+\beta\right)\right|^{2} d \beta\right)^{1 / 2}\left(\int_{\mathcal{N}}|I(\beta)|^{2} d \beta\right)^{1 / 2} \\
\leq & \left(\sum_{q \leq Q_{1}} \sum_{a(q)}^{*} \int_{\mathcal{N}}\left|S\left(\frac{a}{q}+\beta\right)\right|^{2} d \beta\right)^{1 / 2} \\
& \times\left(\sum_{q \leq Q_{1}} \sum_{a(q)}^{*} \frac{\mu^{2}(q)}{\phi^{2}(q)} \int_{\mathcal{N}}|I(\beta)|^{2} d \beta\right)^{1 / 2} \\
\ll & \left(\sum_{q \leq Q} \sum_{a(q)}^{*} \int_{\mathcal{M}_{Q}(q, a)}|S(\alpha)|^{2} d \alpha\right)^{1 / 2} \\
& \times\left(\sum_{q \leq Q_{1}} \frac{\mu^{2}(q)}{\phi(q)} \int_{1 /(2 q Q)}^{1 /(q Q)} \frac{1}{\beta^{2}} d \beta\right)^{1 / 2} \\
\ll & \left(\int_{0}^{1}|S(\alpha)|^{2} d \alpha\right)^{1 / 2}\left(\sum_{q \leq Q_{1}} \frac{q Q}{\phi(q)}\right)^{1 / 2} \\
\ll & \sqrt{Q_{1} Q N \log (2 N)} \ll N \sqrt{\log (2 N)} .
\end{aligned}
$$

For $S_{5}$ we have, by Lemma 3 ,

$$
\begin{aligned}
S_{5}= & \sum_{q \leq Q_{1}} \frac{\mu(q)}{\phi(q)} \int_{1 /(2 q Q)}^{1-1 /(2 q Q)}\left(\sum_{a(q)}^{*} S\left(\frac{a}{q}+\beta\right)\right) \overline{I(\beta)} d \beta \\
= & \sum_{q \leq Q_{1}} \frac{\mu^{2}(q)}{\phi(q)} \int_{1 /(2 q Q)}^{1-1 /(2 q Q)} S(\beta) \overline{I(\beta)} d \beta \\
& +O\left(\log ^{2}(2 N) \sum_{q \leq Q_{1}} \mu^{2}(q) \int_{1 /(2 q Q)}^{1-1 /(2 q Q)}|I(\beta)| d \beta\right) \\
\ll & \sum_{q \leq Q_{1}} \frac{\mu^{2}(q)}{\phi(q)}\left(\int_{1 /(2 q Q)}^{1-1 /(2 q Q)}|S(\beta)|^{2} d \beta\right)^{1 / 2}\left(\int_{1 /(2 q Q)}^{1-1 /(2 q Q)}|I(\beta)|^{2} d \beta\right)^{1 / 2} \\
& +O\left(\log ^{2}(2 N) \sum_{q \leq Q_{1}} \mu^{2}(q) \int_{1 /(2 q Q)}^{1 / 2} \frac{d \beta}{\beta}\right)
\end{aligned}
$$




$$
\begin{aligned}
& \ll \sum_{q \leq Q_{1}} \frac{\mu^{2}(q)}{\phi(q)}\left(\int_{0}^{1}|S(\alpha)|^{2} d \alpha\right)^{1 / 2}\left(\int_{1 /(2 q Q)}^{1 / 2} \frac{d \beta}{\beta^{2}}\right)^{1 / 2}+O\left(Q_{1} \log ^{3}(2 N)\right) \\
& \ll \sqrt{N Q Q_{1} \log (2 N)}+O\left(N^{1 / 2} \log ^{3}(2 N)\right) \ll N \sqrt{\log (2 N)} .
\end{aligned}
$$

Finally we return to $S_{2}$. For $Q \leq N^{1 / 2}$ we have $S_{2}=0$. Assuming $Q>N^{1 / 2}$ we have $Q_{1}=N / Q$ and

$$
\begin{aligned}
S_{2}= & \sum_{Q_{1}<q \leq Q} \frac{\mu(q)}{\phi(q)} \sum_{a(q)}^{*} \int_{\theta_{Q}(q, a)} S\left(\frac{a}{q}+\beta\right) \overline{I(\beta)} d \beta \\
\ll & \sum_{Q_{1}<q \leq Q} \frac{\mu^{2}(q)}{\phi(q)} \sum_{a(q)}^{*}\left(\int_{\theta_{Q}(q, a)}\left|S\left(\frac{a}{q}+\beta\right)\right|^{2} d \beta\right)^{1 / 2} \\
& \times\left(\int_{\theta_{Q}(q, a)}|I(\beta)|^{2} d \beta\right)^{1 / 2} \\
\ll & \left(\sum_{q \leq Q} \sum_{a(q)}^{*} \int_{\mathcal{M}_{Q}(q, a)}|S(\alpha)|^{2} d \alpha\right)^{1 / 2} \\
& \times\left(\sum_{Q_{1}<q \leq Q} \sum_{a(q)}^{*} \frac{\mu^{2}(q)}{\phi^{2}(q)} \int_{-1 /(q Q)}^{1 /(q Q)}|I(\beta)|^{2} d \beta\right)^{1 / 2} \\
\ll & \left(\int_{0}^{1}|S(\alpha)|^{2} d \alpha\right)^{1 / 2}\left(\sum_{Q_{1}<q} \frac{\mu^{2}(q) N^{2}}{q \phi(q) Q}\right)^{1 / 2} \\
\ll & \sqrt{\frac{N^{3} \log (2 N)}{Q Q_{1}}}=N \sqrt{\log (2 N)} .
\end{aligned}
$$

Lemma 4 now follows from (4.3) through (4.9).

5. Proof of the Theorem. As a simple consequence of Lemma 4 we can determine how closely $J_{Q}(\alpha)$ approximates $S(\alpha)$ in $L^{2}$.

Lemma 5. For $1 \leq Q \leq N$ and $Q_{1}=\min (Q, N / Q)$, we have

$$
\int_{0}^{1}\left|S(\alpha)-J_{Q}(\alpha)\right|^{2} d \alpha=N \log \left(N / Q_{1}\right)+O(N \sqrt{\log (2 N)}) .
$$

Pr o of. On multiplying out we find the left hand side of (5.1) is

$$
\int_{0}^{1}|S(\alpha)|^{2} d \alpha-2 \Re \int_{0}^{1} S(\alpha) \overline{J_{Q}(\alpha)} d \alpha+\int_{0}^{1}\left|J_{Q}(\alpha)\right|^{2} d \alpha .
$$

By (1.2), Lemma 2, and Lemma 4 the result follows. 
Proof of the Theorem. By Cauchy-Schwarz we have

$$
\begin{aligned}
\int_{0}^{1}\left|S(\alpha)-J_{Q}(\alpha)\right| d \alpha & \leq\left(\int_{0}^{1}\left|S(\alpha)-J_{Q}(\alpha)\right|^{2} d \alpha\right)^{1 / 2} \\
& =\sqrt{N \log \left(N / Q_{1}\right)}+O\left(N^{1 / 2} \log ^{1 / 4}(2 N)\right) .
\end{aligned}
$$

By the reverse triangle inequality and Lemma 1 the left hand side of (5.2) is

$$
\geq \int_{0}^{1}|S(\alpha)| d \alpha-\int_{0}^{1}\left|J_{Q}(\alpha)\right| d \alpha \geq \int_{0}^{1}|S(\alpha)| d \alpha-O\left(N^{1 / 2}\right) .
$$

The Theorem now follows on taking $Q=Q_{1}=N^{1 / 2}$ in (5.2).

\section{References}

[1] G. H. Hardy and J. E. Littlewood, Note on Messrs. Shah and Wilson's paper entitled: 'On an empirical formula connected with Goldbach's theorem', Proc. Cambridge Philos. Soc. 19 (1919), 245-254.

[2] - - - Some problems of "Partitio Numerorum": III. On the expression of a number as a sum of primes, Acta Math. 44 (1923), 1-70.

[3] R. C. Vaughan, The $L^{1}$ mean of exponential sums over primes, Bull. London Math. Soc. 20 (1988), 121-123.

[4] D. R. Ward, Some series involving Euler's function, J. London Math. Soc. 2 (1927), 210-214.

Department of Mathematics and Computer Science

San Jose State University

San Jose, CA 95192, U.S.A.

E-mail: goldston@mathcs.sjsu.edu 